\title{
SURGIMIENTO Y DESARROLLO DE COSECHADORAS DE CEREALES. CASO DE ESTUDIO CUBA.
}

Idalberto Macías, Antonio Barrera, Lenni Ramírez, Mercedes Arzube. 


\title{
Surgimiento y desarrollo de cosechadoras de cereales. Caso de estudio Cuba
}

\section{Development and development of cereal harvesting. Case study Cuba}

\author{
Idalberto Macías Socarrás; Antonio Luis Barrera Amat; Lenni Ramírez Flores; Mercedes Arzube Mayorga \\ Facultad de Ciencias Agrarias \\ Universidad Estatal Península de Santa Elena (UPSE). \\ Campus La Libertad, vía principal Santa Elena - La Libertad La Libertad-Ecuador. \\ imacias@upse.edu.ec
}

\begin{abstract}
Resumen
En el presente trabajo se hace una reseña histórica acerca del surgimiento y desarrollo de las cosechadoras de cereales en el mundo, su introducción en Cuba, así como el papel que se ha jugado en el desarrollo de estas máquinas, en este artículo se tratan los equipos empleados para la recolección de cultivos aprovechados por sus granos, fundamentalmente los cereales (trigo, cebada, avena, centeno, maíz, sorgo, arroz), Adicionalmente, se explicarán aspectos de las cosechadoras: la evolución histórica de la recolección de cereales y, finalmente, los elementos básicos que forman parte de estas máquinas, modo de funcionamiento, regulación y pérdidas. Además, de exponer los elementos y componentes más habituales de las cosechadoras, se incluye especialmente las novedades y avances técnicos que se empiezan a implantar en las cosechadoras, como pueden ser los sistemas de trilla forzada, la separación rotativa, los sensores electrónicos de rendimiento.
\end{abstract}

Palabras clave: Cosechadoras de cereales, recolección, regulaciones, pérdidas.

\begin{abstract}
In this paper, a historical review is made of the emergence and development of cereal harvesters in the world, their introduction in Cuba, as well as the role played in the development of these machines, this article deals with the equipment (Cereals, wheat, barley, oats, rye, maize, sorghum, rice), aspects of harvesters will also be explained: the historical evolution of cereal harvesting and, finally, The basic elements that are part of these machines, mode of operation, regulation and losses. In addition, to exposing the most common elements and components of combine harvesters, special mention is made of the novelties and technical advances that are beginning to be introduced in harvesters, such as forced threshing systems, rotary separation, and electronic performance sensors.
\end{abstract}

Key word: Cereal harvesters, harvesting, regulations, losses.

\section{Introducción.}

Desde épocas remotas el hombre siempre buscó la manera de hacer más fácil su trabajo en la agricultura; 
con el transcurso del tiempo se fueron perfeccionando los instrumentos de trabajo y se comenzaron a mecanizar los mismos.

La cosecha representa para el agricultor la culminación de largas y fatigosas operaciones; por la cual él mismo puede recoger lo que con su trabajo ha sabido obtener de la tierra. Esta operación presupone de un largo tiempo y se ha buscado la manera de hacerla lo más fácil y rápida posible; procurando poner al seguro la misma, con la mayor brevedad posible. Con el uso de la maquinaria, además de reducir el tiempo empleado, la fatiga y la necesidad de mano de obra, se ha aumentado el rendimiento, ya que en su conjunto se han reducido las diversas operaciones.

En la actualidad son muchos los modelos y marcas de cosechadoras de cereales que existen en el mercado, compuestas generalmente por elementos muy similares, que varían poco de un fabricante a otro. En los últimos años se ha experimentado una importante evolución en el mundo de las cosechadoras, adaptándose correctamente a las condiciones y características de recolección de un amplio abanico de cultivos. Entre ellos, los que se recogen con este tipo de maquinaria se destacan los cereales (trigo, cebada, avena, centeno, maíz, sorgo, arroz, etc.), oleaginosas como girasol, colza, soja, y las leguminosas para grano (lentejas, porotos, etc.). Además se cosecha una amplia variedad de semillas forrajeras como alfalfa, tréboles, y gramíneas.

En Cuba se introducen en la década de 1950; precisamente en 1956 hace su entrada la JOHN DEERE de fabricación norteamericana, las mismas fueron evolucionando con el paso de las décadas, sin embargo es necesario organizar cronológicamente los diferentes periodos en que son introducidas las nuevas máquinas, lo que serviría como un material de estudio para los profesionales del campo de la mecanización.

Otro aporte de este trabajo es que se realiza un estudio de forma separada para una mejor comprensión de la plataforma de corte, elementos de separación y limpia, elementos de trilla, los nuevos sistemas de separación alternativos, dispositivos auxiliares y sistemas automáticos, pérdidas y regulaciones, constituyendo este un aporte significativo para contrarrestar las pérdidas que ocurren durante el proceso.

Por tal motivo, en el presente trabajo tiene como objetivo determinar los aspectos fundamentales que dieron origen a las cosechadoras de cereales actuales, cómo se introdujeron en Cuba y el papel que se ha jugado en el desarrollo de estas máquinas, igualmente se tocarán los elementos básicos que forman parte de estas máquinas, modo de funcionamiento, regulación y pérdidas y tendencias actuales en la fabricación.

\subsection{Instrumentos que dieron origen al surgimiento de las cosechadoras de cereales actuales}

Antes del surgimiento de las máquinas cosechadoras de cereales el arroz era cortado a mano; parece que en el origen (edad de piedra) los primeros agricultores usaban piedras filosas que posteriormente reemplazaron con cuchillas algo encorvadas, dando origen a la hoz. La hoz es un instrumento muy antiguo (es usado desde los tiempos bíblicos); en épocas de la antigua Roma ya se usaban hoces análogas a las actuales. Angladette, (1969)

Aunque tan sencilla en su aspecto, pueden ser objeto de algunas interesantes consideraciones de orden técnico-mecánico, por lo que se pueden distinguir de tres clases o formas. Ibáñez, J; González-Urquijo J; Gibaja J, (2013).

$>$ La de lámina ancha, filo liso y con curvatura próxima a la circular.

$>$ La de hoja angosta, filo aserrado y curvatura tipo espiral.

$>$ La tipo norteamericano, de lámina ancha y filo ligeramente curvo y ondulado.

Está formada por una hoja encorvada sujeta a un cabo de madera, la hoja es de acero, el filo cortante por lo general es liso, y se afila con piedras de agua, aunque las actuales son autoafilantes. Un obrero algo entrenado en el manejo de la misma puede cosechar en un día de trabajo una superficie que varía entre los 1000 y 1500 $\mathrm{m}^{2}$.

Luego surgen las guadañas, que es un instrumento que permite realizar el trabajo con un rendimiento mayor; con este instrumento un obrero puede cosechar en un día de trabajo una superficie de $2500 \mathrm{~m}^{2}$. Actualmente, en muchos lugares del mundo son utilizadas las hoces y las guadañas por productores pequeños.

En la antigüedad, los primitivos usaban para el desgrane o trilla de los cereales el pie, también utilizaron animales como asnos, mulos, caballos y otros; además, muchos cosechadores utilizaron troncos de madera, a manera de pisón.

En Vietnam y en Camboya se trilló con rodillos de piedras ( $0.8 \mathrm{~m}$ de largo y de 0.22 a $0.25 \mathrm{~m}$ de diámetro); también se utilizaron series de discos con muescas de 0.3 a $0.4 \mathrm{~m}$ de diámetros alternados, es el principio del "norag" de Egipto. El desgrane es satisfactorio con estas máquinas, pero tiene el inconveniente de que las roturas en los granos sean excesivas. Angladette, (1969)

Muchos fueron los intentos por desarrollar la trilla, pero no es hasta 1786 en que el escocés Andrew Meckle construyó la primera trilladora que estableció los principios mecánicos de las trilladoras modernas. Aquellas máquinas tenían como pieza principal de trabajo un cilindro de listines rayados en combinación con un cóncavo también con listones rayados, como en las máquinas actuales. INRA, (1975)

Pocos años más tardes el italiano Domérico Fortuna (1794), quizás desconociendo aquellas, ideó una máquina análoga destinada ya especialmente para la trilla del arroz. 
Los perfeccionamientos ulteriores de estas primeras máquinas no fueron por cierto muy rápidos; en Norteamérica, las trillas fueron conocidas alrededor de 1815; en 1835 ellas habían alcanzado en Europa cierto grado de adelanto y su empleo se iba generalizando.

En la década de 1830 muchos granos se trillaban aún con rodillos rústicos de madera y caballos. Diversos inventores obtuvieron patente, pero la concedida a Hirán A. y John A. Pitts el 29 de diciembre de 1837 fue realmente el comienzo de la trilladora, esta máquina era accionada por caballos. En 1840 había, en Estados Unidos, fábricas de trilladoras de cierta importancia; la fábrica de trilladoras CASE comenzó en 1844 en Racine, Wisconsin. Hacia 1900 estas máquinas estaban equipadas con alimentadores automáticos, cortadores de cuchilla, pesadoras y sopladores para apilar la paja o tubos lanzapajas.

El uso del viento para la limpieza del grano es muy antiguo teniendo su origen muchos siglos antes de nuestra era. En esa época se colocaba el grano a limpiar en cestas profundas y se dejaba caer desde una altura relativamente grande con suave descarga al viento; en ciertas circunstancias el operador se puede subir en un andamiaje ligero para hacer caer el grano desde una altura mayor; este método permite eliminar las ligeras impurezas del grano (polvo, pajas menudas, ligeros residuos vegetales, etc). Para separar al grano de las impurezas el método más usado es el arneo con una cesta plana que por sacudidas sucesivas permite su limpieza. Conti, (1950). Angladette, (1969); Auge, (1976)

En Filipinas se crea un equipo combinado que permite la trilla por pisoteo sobre un andamiaje enrejado por donde cae el grano, aventado en el curso de su caída por la acción del viento. Así, se pusieron a punto diferentes instrumentos más o menos complicados para realizar el aventado, no dando resultados satisfactorios. Es entonces cuando surge el ventilador artesanal, muy rústico por cierto. Estos ventiladores eran construidos de madera y de tejidos accionados primeramente a mano y luego por manivelas. Poco a poco se le fueron adaptando dispositivos como engranajes, poleas y correas que multiplicaban entre cuatro y cinco veces la velocidad de la manivela.

Estos ventiladores se fueron perfeccionando $\mathrm{y}$ combinando con trilladoras, cribas y zarandas, dando origen de esta forma a las máquinas cosechadoras clásicas más conocidas, que con distintas características constructivas consiguen la misma finalidad.

\subsection{Surgimiento y desarrollo de las cosechadoras de cereales.}

Las máquinas para cosechar cereales (en particular el trigo) tienen una historia muy antigua, más que las de cosechar forrajes (estas últimas se derivaron de aquellas con oportunas modificaciones). Refiere la historia que en la época de Julio Cesar, los Galos ya usaban una verdadera espigadora primitiva que consistía en un carro de madera con bordes en forma de peines tirado por animales. Conti, (1950). Angladette, (1969); Auge, (1976)

Las primitivas iniciativas no tuvieron por cierto el éxito deseado; tal es así, que la máquina quedó olvidada por muchos siglos y no volvemos a encontrar en la literatura geórgica nada al respecto hasta principios del siglo XIX, época en que hubo un verdadero despertar en la mecánica aplicada a la agricultura. Aparecen las segadoras de Gladstone (1806), la de Salomón (1808) y la de Smith (1810) cuyos órganos de corte eran fundamentados sobre principios muy distintos a los actuales y movidas por tracción animal. Conti, (1950); Ochoa (1997).

Las mayores dificultades se hallaron en la construcción del aparato cortante, después de ensayar cuchillas formadas por guadañas animadas por un movimiento de rotación, se ensayaron discos y sierras circulares, pero pronto todos estos dispositivos fueron olvidados y el problema no entro en su verdadera solución práctica hasta 1827 , año en que el cura escocés Patrick Bell, hizo funcionar su primera segadora, cuyo órgano cortante estaba formado por dientes fijos y sierra de movimiento alternativo. Es esta máquina la que debe considerarse como el prototipo de la segadora; sobre ella se moldearon las muchas otras construidas posteriormente en Europa y sobre todo en los Estados Unidos de Norteamérica, máquinas que han alcanzado en pocos años una perfección verdaderamente admirable. Ibáñez, J; González-Urquijo J; Gibaja J, (2013).

Entre los muchos que colaboraron en esta tarea están los nombres de McCormick, Deerin, Hussey, Burgess, Walter Wood, Counier, entre otros, los que en diferentes tiempos y en distintos países contribuyeron a perfeccionar esta máquina que tantos beneficios aportó al abaratamiento de la producción en el cultivo extensivo de los cereales.

En 1822 fue cuando se intentó por primera vez construir una máquina de tracción animal destinada a cortar hierba, pero no es hasta 1840 que se introdujo de forma definitiva, apareciendo en los Estados Unidos la primera barra de corte.

Obed Hussey obtuvo una patente para segadora en 1833, y McCormick alegaba haber hecho demostraciones de su primera segadora tirada por caballos en 1831, pero no obtuvo la patente hasta 1834 . Según los historiadores esta máquina desempeñó un papel decisivo en la guerra de secesión de los Estados Unidos ya que permitió a los estados del norte, más industrializados, hacer frente a la recolección de cereales con menor cantidad de mano de obra, sin tener que depender de los estados del sur, como sucedía al momento de comenzar la contienda. Alrededor de 1850 se introduce una plataforma para el atado manual de las gavillas, la segadora agavilladora aparece en 1854. El primer mecanismo para atar con alambre aparece en 1873 y los atadores de hilo fueron introducidos en 1880, pero no fue hasta 1892 en que Apleby obtuvo una patente de anudador para hilo. Ochoa (1997). 
Las atadoras tiradas por caballos estaban accionadas por ruedas. Hacia 1920 se montaron motores auxiliares en algunas de ellas, y se introdujo la atadora accionada por el árbol de toma de potencia de los tractores, que ya habían surgido desde 1839.

Lo mismo que puede decirse que el tractor es el rey de la mecanización agraria, a la cosechadora puede asignársele la denominación de reina, por realizar una multiplicidad de acciones simultáneamente (siega, trilla, aventado y clasificación de semillas, etc), ahorrando una cantidad muy grande de mano de obra.

En 1828 se dio a Simule Lane una patente para lo que se denominó segadora-trilladora combinada. Pero el comienzo real de la máquina para recolectar, trillar y limpiar fue cuando A.Y. Moore y sus colaboradores de Kalamazoo, Michingan, obtuvieron una patente en 1835.

En 1854 ya se cosecharon 243 hectáreas de trigo en Alamade Country, California, pero el método no se difundió realmente en California hasta 1880, aproximadamente. Uno de los primeros fabricantes de las cosechadoras tiradas por caballos y accionadas por ruedas fue la Stockton Combined Harvester and Agricultural Works of California. Ochoa R. (1997).

Las cosechadoras accionadas por tractor de vapor se introdujeron hacia los años 1880. Algunas de estas máquinas estaban equipadas con un mecanismo segador de $13 \mathrm{~m}$, cosechando, según se dice, de 36 a 50 ha/día. En 1910, con la sustitución del peine por la cuchilla o sierra, se marcó un gran adelanto en la construcción de estos equipos; desde entonces el perfeccionamiento de dichas máquinas fue constante y su empleo se fue extendiendo especialmente en los países de cultivos extensivos con gran beneficio económico para los agricultores.

En un principio, los constructores norteamericanos se dedicaron a fabricar cosechadoras de gran tamaño. Por los años 1912 al 1914, aparecieron las cosechadoras Holt con cuchillas de 18 a 20 y hasta 30 pies (5.5 a 6 y hasta $9.144 \mathrm{~m}$ ), pero pronto se vio que esto no resultaba práctico y luego de muchos ensayos se fijó una medida mas o menos normal de 12 pies (3.6576 m), extensible en algunos casos a 14 pies $(4.2672 \mathrm{~m})$ y a lo sumo 16 pies $(4.8718 \mathrm{~m})$ para cereales limpios y de muy pocas pajas. De Toro, A; Gunnarsson, C; Lundin, G; Jonsson, N. (2012).

Las cosechadoras accionadas por tractor de gasolina se introdujeron en amplia escala en las zonas trigueras del centro del este de los Estados Unidos como consecuencia de la escasez de la mano de obra durante la primera guerra mundial o aproximadamente hacia 1916. Las cosechadoras se introdujeron por primera vez en el nordeste de Texas en 1919, época en que se utilizaron siete máquinas de este tipo. Conti, (1950).

Las cosechadoras autopropulsadas se introdujeron comercialmente alrededor de 1938. Ya en el año 1941 el mercado americano ofrecía en venta 59 modelos de cosechadoras, de las cuales 20 eran con cilindro de dientes, 29 con listones de acero y 10 con listones de goma endurecida. El $20 \%$ de estas máquinas son accionadas por la toma de potencia del tractor y el resto con motor propio, llegando a dividirse en cosechadoras remolcadas o de arrastre y las autopropulsadas. De Toro, A; Gunnarsson, C; Lundin, G; Jonsson, N. (2012).

En la actualidad las autopropulsadas se están produciendo en grandes cantidades y por diferentes empresas; dentro de los principales fabricantes de estas máquinas se encuentran la JOHN-DEERE, CLASS y NEW HOLLAND.

\section{3.- Las máquinas cosechadoras de cereales en Cuba.}

En Cuba, las máquinas cosechadoras de cereales se emplean fundamentalmente para la cosecha del arroz; por ser éste el cereal que más se cultiva. Estas máquinas se introducen en la década de 1950; precisamente en 1956 hace su entrada la JOHN DEERE de fabricación norteamericana.

A partir delos primeros años de la década del sesenta, son introducidas diferentes marcas de cosechadoras; al principio, fundamentalmente, procedían del campo socialista en especial de la antigua URSS.

En la década del 60 entran las cosechadoras polacas VISTULAS, las cosechadoras soviéticas CKG-3, CKG4 (NIVA) y la CKG-5 (KOLOS), y la cosechadora italiana LAVERDA M120.

En los años 70, son traídas nuevas marcas de cosechadoras italianas, estas son la LAVERDA M132 y FIAT 3450, así como las marcas alemanas WEIMAR E175 y WEIMAR E-117.

Ya en la década del 80, se introducen dos marcas de cosechadoras del campo socialista, una que es la alemana WEIMAR E-514 y la E-1200 de fabricación soviética; ocupando Italia el papel principal en el suministro de estas máquinas con la LAVERDA 3500 y LAVERDA 3550.

En la década del 90 se introduce la cosechadora INTERNATIONAL HARVESTER modelo IDEAL 9075 de fabricación brasileña. En el año 1996 entra al país una nueva marca de cosechadoras de cereales, la LAVERDA L517M y la LAVERDA L517H de la generación de NEW HOLLAND, de esta misma generación entra en 1997 la LAVERDA L 624 FIAT.

En el desarrollo de estos equipos, juega un papel importante la fábrica de "Héroes del 26 de Julio" de la provincia de Holguín, donde se fabricaron las primeras cosechadoras cubanas denominadas CUBAR-90 e IMPAG-411 en cooperación con la firma italiana FIATAGRI. Hay que señalar que la CUBAR-90 sirvió de prototipo para pruebas y posterior construcción de la IMPAG-411, muchos de estos modelos se pueden ver en los campos arroceros cubanos.

Esto no quiere decir que las cosechadoras de cereales han sido estudiadas con profundidad en Cuba; muchos de los agregados, sistemas y conjuntos no se han investigado a cabalidad y sobre estos se puede influir positivamente. Actualmente, en la Empresa de Implementos Agrícolas Héroes del 26 de julio en la 
provincia de Holguín se continúan realizando pruebas para la mejora de los diferentes sistemas, para elevar su fiabilidad técnica y operativa.

\section{4.- Plataforma de corte}

La capacidad de trabajo de una cosechadora viene determinada teóricamente por la anchura del corte, aunque en realidad el factor limitante es la cantidad de paja que pueden trabajar los sacudidores. Todos los dispositivos directamente relacionados con el corte se agrupan en el elemento denominado "plataforma" o cabezal. En las máquinas de gran tamaño debe desmontarse y cambiarse de posición para su transporte (remolcado), aunque existen modelos que evitan estas operaciones al disponer de plegado mecánico de la plataforma. Ortiz-Cañavate (2012)

Los elementos de la plataforma de corte son: barra de corte molinete y órganos de alimentación. Mientras la barra de corte siega el tallo, el molinete abate la parte superior de la mies, asegurando su caída hacia el tomillo sinfín embocador, que se encargará de conducirla hacía la boca de alimentación y desde allí, por medio de una banda elevadora interior, llegará al mecanismo de trilla. La barra de corte puede regularse en altura, así como equiparse con dedos auxiliares levanta mieses. A ambos lados de la plataforma de corte van unos divisores de mies en forma de cuña. El molinete, por su parte, admite varias regulaciones: velocidad de giro y posiciones de altura y en avance respecto a la cuchilla de corte. OrtizCañavate J. et all. (2012)

El molinete se construía antiguamente con 4, 6 u 8 tablas dispuestas radialmente, de una forma comparable a las segadoras-atadoras. Sin embargo, el choque con las espigas ocasionaba pérdidas importantes por desgrane tanto más cuanto más baja era la humedad de la espiga, sustituyéndose por las formas actuales con dedos dotados de orientación fija durante toda la vuelta, lo cual se consigue fácilmente mediante paralelogramos articulados. Valero (2007).

Los órganos de alimentación están constituidos por el tornillo sinfín embocador y por la banda elevadora. El primero tiene tres partes diferenciadas; las dos extremas tienen el paso en sentido opuesto, con objeto de obligar a la mies a ir hacia la parte central, en donde se la empuja en dirección a la banda elevadora con ayuda de unos dedos retráctiles. Esta banda elevadora está constituida por dos o tres cadenas unidas mediante barras angulares de chapa de borde dentado, que empujan a la mies a través de la rampa inclinada que asciende hasta el cilindro desgranador. La banda elevadora está en posición flotante y la tensión del rodillo inferior la regula un muelle, con el fin de conseguir un mejor ajuste a la mies. Valero (2007).

\subsection{Elementos de trilla}

La operación que realizan los elementos del sistema de trilla de una cosechadora es la separación del grano de la espiga. Los órganos de trilla de una cosechadora convencional son el cilindro desgranador y el cóncavo, con los que se separa, es decir, se trilla, alrededor del $90 \%$ de los granos. Este número también se conoce como "eficacia de trilla". Gil (1994); Valero (2007).

Para poder realizar la trilla se necesita que la humedad del grano sea inferior al 20-22\%, mientras que para almacenarlo es conveniente que la humedad no pase del 15\%. Ortiz-Cañavate J. et all. (2012)

Existen básicamente dos tipos de cilindros desgranadores:

- De dientes o dedos.

- De barras.

El cilindro desgranador es un órgano de gran masa que gira a una elevada velocidad, por lo que es necesario equilibrarlo no sólo estática, sino también dinámicamente. Ortiz-Cañavate J. et all. (2012)

Para proteger el cilindro desgranador del impacto con piedras, suele disponerse un depósito previo antes del cóncavo en el que quedan retenidos las piedras $u$ objetos extraños antes de que puedan dañar el sistema de trilla. Detrás del cilindro desgranador va un cilindro batidor, también llamado lanza pajas o molinete expulsor, que ayuda a pasar la paja a los sacudidores. Valero (2007); Ortiz-Cañavate J. et all. (2012)

\subsection{Elementos de separación y limpia}

Según Gil (1994) las funciones que realiza el sistema de limpia de una cosechadora son:

a) La separación del grano de la paja.

b) La limpieza del grano o separación del tamo, envolturas, polvo y semillas extrañas.

Los órganos de separación y limpia de una cosechadora convencional son:

- Sacudidores: criba única o conjunto de cribas con amplios agujeros y con movimiento de vaivén, que sirve para separar el resto del grano $(10 \%)$ lo que queda entre la paja.

- Caja de limpia, con una o varias cribas con movimiento oscilante para separar el grano de la paja corta y del tamo, que son arrastrados por la corriente del ventilador.

Ortiz-Cañavate J. et all. (2012) indica que por analogía los elementos de las antiguas trilladoras, los sacudidores son denominados también saca pajas o zarandones, y el fondo inclinado de la caja de cribas que recoge los residuos de las espigas.

Valero (2007) resalta que el sacudidor convencional esté constituido por un conjunto de rejillas calibradas que permiten el paso del grano y la paja corta. Puede estar formado por un solo elemento o por varios elementos dentados en forma de rampas oscilatorias accionadas por un cigüeñal, cuyo radio de muñequilla varía entre 4 y $10 \mathrm{~cm}$, desplazándose adelante y atrás a un ritmo de 200 a 250 oscilaciones por minuto.

\subsection{Los nuevos sistemas de separación alternativos}

La corriente mundial en la fabricación de cosechadoras de cereales se inclina hacia la el sistema de separación rotativa de flujo axial, es el que sustituye el cilindro 
desgranador típico transversal y todo el sistema de limpia por una pareja de cilindros desgranadores paralelos (uno o dos, según tipos) longitudinales a lo largo de la cosechadora y de grandes dimensiones, que giran dentro de otros dos cilindros fijos concéntricos. Valero C. (2004), Valero C. (2007).

Cada rotor cilíndrico longitudinal posee las siguientes partes:

- Aspirador de paletas helicoidales en su extremo anterior para alimentarla mies.

- Conjunto de barras o dedos trilladores dispuestos helicoidales y longitudinalmente en su primera mitad por su superficie, en ocasiones con aristas o estrías.

- Conjunto de dedos más espaciados en su segunda mitad o barras lisas helicoidales, cuya misión es la de separar el grano de paja previamente trillado.

Este sistema alcanza mayor eficacia cuando la velocidad periférica del rotor (o rotores) se sitúa aproximadamente en los $25 \mathrm{~m} / \mathrm{s}$ para cultivos de cereal del tipo trigo y cebada. Se han observado mejoras de rendimiento en cosechadoras de maíz, pero se muestra similar al sistema clásico en la recolección de cereales de invierno. Al ser más corta la cosechadora resulta más maniobrable y su mecánica más sencilla. Además, con este sistema, los procesos de trilla y separación se realizan en la mitad de tiempo que en una cosechadora convencional. Valero C. (2004), Valero C. (2007).

Basados en este sistema de separación de flujo axial existen dos tipos de máquinas:

a) De rotor único

Dicho rotor tiene un diámetro de $75 \mathrm{~cm}$ y una longitud de $270 \mathrm{~cm}$, presentando una superficie de trilla y separación de $2 \mathrm{~m}^{2}$. La velocidad de rotación puede regularse entre 280 y $1.040 \mathrm{r} / \mathrm{min}$.

b) De doble rotor

Se compone de dos rotores paralelos que giran en sentido inverso de unos $220 \mathrm{~cm}$ de longitud y unos 45 $\mathrm{cm}$ de diámetro cada uno, que giran a una velocidad regulable entre 580 y $1.320 \mathrm{r} / \mathrm{min}$. La superficie de trilla y separación es también de $\mathrm{m}^{2}$

\subsection{Dispositivos auxiliares $y \quad$ sistemas automáticos}

La plataforma de corte es un elemento de la cosechadora en el que se forman con frecuencia atascos de producto, que paralizan la labor y obligan al conductor a operar sobre el punto de atasco. Por ello, es frecuente que las plataformas actuales incorporen un sistema de inversión del giro desde la cabina para desatascar automáticamente los órganos de alimentación. Además, para facilitar su transporte y maniobrabilidad existen plataformas de corte plegables. Hancock, M; Duffield, S; Boyle, B; Wilson, J. (2015).

Autores como Valero (2007) y Ortiz-Cañavate J. et all. (2012) coinciden en plantear que durante el trabajo en terrenos desiguales o en pendiente, afecta tanto a la plataforma de alimentación como a la separación y limpia. Respecto a la primera, mediante los sistemas electrónicos de autoflotación, la plataforma de corte se mantiene paralela a la superficie del terreno longitudinal y transversalmente, produciendo una altura de rastrojo uniforme. El sistema incluye cilindros hidráulicos de doble efecto y sensores ultrasónicos instalados en la parte inferior del cabezal que miden constantemente la distancia al suelo, de forma que el cabezal sube o baja automáticamente auxiliado por unos patines de presión. Hancock, M; Duffield, S; Boyle, B; Wilson, J. (2015).

Existen también cosechadoras autonivelantes para terrenos con pendiente de hasta el $45 \%$. La nivelación se consigue mediante unos cilindros hidráulicos que modifican la posición de las ruedas con respecto al cuerpo de la máquina, a la vez que oscila el cabezal. Ochoa (1997), Gil (1994).

Las nuevas cosechadoras de cereales buscan aumentar el confort del conductor. Las cabinas son centros de trabajo desde los que se pueden controlar todos los elementos de la máquina de forma fácil y cómoda. Todos los fabricantes incorporan monitores digitales o pilotos analógicos que informan al conductor de las operaciones que la máquina está realizando, de los regímenes de funcionamiento de los elementos y de los posibles problemas o averías. Mediante mandos multifunción y pulsadores varios, el operador puede regular el cabezal, el sistema de trilla, los sacudidores y cribas, el funcionamiento del ventilador de limpia, la descarga. Valero (2007) y Ortiz-Cañavate J. et all. (2012)

\subsection{Pérdidas y regulaciones}

Autores como García, (1989); Ochoa (1997); Gil (1994); Valero (1997); Miranda et al, (2011) y Pozzolo et al, (2015) coinciden en que las pérdidas en las cosechadoras pueden ocurrir:

- Antes de la recolección, por dehiscencia natural de las espigas.

- En la plataforma de corte y en el molinete.

- En el cilindro desgranador y cóncavo: el grano se parte, no se trilla suficiente.

- En los sacudidores: el grano se pierde con la paja.

- En las cribas: el grano se pierde con el tamo.

Modernamente las cosechadoras disponen de unos detectores automáticos de pérdidas, gracias a unos sensores electrónicos que se sitúan Inmediatamente detrás de los sacudidores y de las cribas. Su misión es detectar la cantidad de grano que se pierde por dichos lugares, transmitiendo una señal eléctrica a un indicador situado en el puesto de conducción. De este modo, el operario puede advertir las irregularidades del funcionamiento y trabajar con la mínima pérdida de grano. Hancock, M; Duffield, S; Boyle, B; Wilson, J. (2015).

En ocasiones, aunque la cosechadora tenga sensores de pérdidas, es necesario realizar ensayos de campo para obtener resultados precisos. En los ensayos de pérdidas se hacen recuentos del grano caído antes de la cosecha, del que queda debajo de la máquina, se cuenta el grano partido y se recoge con lonas lo que cae 
de los sacudidores y de las cribas. Ortiz-Cañavate J. et all. (2012) y Hancock, M; Duffield, S; Boyle, B; Wilson, J. (2015).

Es muy importante considerar las pérdidas que se producen en las cosechadoras, que pueden representar valores importantes en una explotación (normalmente, $2-6 \%$, pudiendo incluso llegar al 10\%). El nivel de las pérdidas viene muy Influido por las condiciones atmosféricas y por el método de recolección, pero siempre se aprecian claras diferencias a favor de los sistemas más mecanizados como es la cosechadora, donde las pérdidas son mucho menores que con los métodos primitivos. Ortiz-Cañavate J. et all. (2012) y Hancock, M; Duffield, S; Boyle, B; Wilson, J. (2015).

\section{Conclusiones}

1. Dentro de los instrumentos que dieron origen a las cosechadoras de cereales actuales se encuentran la hoz, las guadañas, ventiladores y cribas.

2. La hoz marcó un paso muy importante por ser el primer instrumento que utilizó el hombre para la cosecha de los cereales, su divulgación y uso fue tal que actualmente en muchos lugares del mundo es utilizada por pequeños agricultores.

3. En 1827, hizo funcionar la primera segadora, cuyo órgano cortante estaba formado por dientes fijos y sierra de movimiento alternativo, es ésta máquina la que debe considerarse como el prototipo de la segadora que da origen a las cosechadoras modernas.

4. En Cuba no se han hecho estudios profundos con vista a desarrollar estas máquinas, a pesar de haberse construido algunas de ellas en cooperación con firmas extranjeras.

5. Es fundamental la preparación técnica de los operarios con vistas el manejo correcto de los diferentes componentes de las cosechadoras.

\section{Bibliografía}

[1] Angladette, A. (1969) El Arroz. Barcelona: Editorial Blume.

[2] Arroz INRA. Grupo Nacional del Arroz. La Habana: Editorial Científico-Técnica, 1975.

[3] Auge, R. (1976). Mecánica Agrícola. Madrid: Paraninfo.

[4] Barañao V (1982). Maquinaria Agrícola. Argentina: Buenos Aires.

[5] Bermejo A. (1972). Manual Práctico del Mecánico Agrícola. Madrid: Publicaciones de Capacitación Agraria. Tomo 2.

[6] Conti, M. (1950). Las Máquinas en la Agricultura Moderna. Tratado de Mecánica Agrícola. Buenos Aires: Talleres Gráficos Bartolomé U. Chiesino.
[7] García, A. (1989). Cosechadoras de Cereales: Cosecha de Granos y Semillas. Italia. Editora de la Oficina Regional de la FAO para América Latina y el Caribe. Roma.

[8] MINAGRI. (1999). Instrucciones Técnicas para el Cultivo del Arroz. Dirección del Arroz. Ciudad de la Habana: Editado en el Centro de Información y Documentación Agropecuaria.

[9] Ochoa R. (1997). Metodología de Cálculo de los Parámetros Fundamentales del Sistema de Limpieza de las Cosechadoras de Cereales. Tesis presentada en opción al título de Master en Maquinaria Agrícola. Universidad de Holguín.

[10] Gil J (1994). Tendencias y novedades de las Cosechadoras de Cereales. Vida Rural. (España); No 295.

[11] Valero C. (2007). Cosechadoras de cereal: historia, elementos y funcionamiento. Departamento de Ingeniería Rural UPM. Salvado de: http://oa.upm.es/6375/1/Valero 71.PDF

[12] Ortiz-Cañavate J. et all. (2012). Las máquinas agrícolas y su aplicación. Editorial Mundi-Prensa, 7 ma Edición. Madrid. 467 p.

[13] Valero C. (2004). Sistemas de trilla y separación en cosechadoras de cereal. Departamento de Ingeniería Rural UPM. Salvado de: http://oa.upm.es/6311/1/Valero_48.pdf

[14] Pozzolo O. et al, (2015) Control de Pérdidas en Cosecha de Arroz. Universidad Nacional de Entre Ríos (UNER). Salvado de: http://www.cosechaypostcosecha.org/data/articulo s/cosecha/perdidasCosechaArroz.asp

[15] Miranda A, et al, (2011). Determinación del contenido de impurezas durante la cosecha de arroz. Revista Ciencias Técnicas Agropecuarias vol.20 no.4. ISSN: 2071-0054.

[16] Ibáñez, J; González-Urquijo J; Gibaja J (2013). Discriminating wild vs domestic cereal harvesting micropolish through laser confocal microscopy. Journal of Archaeological Science. Volume 48, August 2014. pp 96-103

[17] De Toro, A; Gunnarsson, C; Lundin, G; Jonsson, N. (2012). Cereal harvesting - strategies and costs under variable weather conditions. Biosystems Engineering. Volume 111, Issue 4, April 2012, pp 429-439

[18] Hancock, M; Duffield, S; Boyle, B; Wilson, J. (2015). The effect of harvest method on cereal stubble use by seed-eating birds in a High Nature Value farming system. Agriculture, Ecosystems \& Environment. Volume 219, 1 March 2016, pp 119 124 